\title{
Palladium-catalyzed cross coupling reactions of 4-bromo-6H-1,2-oxazines
}

\author{
Reinhold Zimmer ${ }^{*}, 1$, Elmar Schmidt ${ }^{2}$, Michal Andrä ${ }^{1}$, Marcel-Antoine Duhs ${ }^{1}$, \\ Igor Linder ${ }^{1}$ and Hans-Ulrich Reissig ${ }^{*}, 1$
}

\author{
Preliminary Communication \\ Address: \\ ${ }^{1}$ Freie Universität Berlin, Institut für Chemie und Biochemie, \\ Takustrasse 3, D-14195 Berlin, Germany and ${ }^{2}$ Technische Universität \\ Dresden, Institut für Organische Chemie, D-01061 Dresden, Germany \\ Email: \\ Reinhold Zimmer* - rzimmer@chemie.fu-berlin.de; \\ Hans-Ulrich Reissig* - hans.reissig@chemie.fu-berlin.de \\ * Corresponding author \\ Keywords: \\ alkyne; halogenation; 1,2-oxazines; palladium catalysis; pyridines
}

Beilstein Journal of Organic Chemistry 2009, 5, No. 44. doi:10.3762/bjoc.5.44

Received: 04 June 2009

Accepted: 28 August 2009

Published: 16 September 2009

Associate Editor: I. Marek

(c) 2009 Zimmer et al; licensee Beilstein-Institut.

License and terms: see end of document.

\begin{abstract}
A number of 4-aryl- and 4-alkynyl-substituted $6 H$-1,2-oxazines 8 and 9 have been prepared in good yields via cross coupling reactions of halogenated precursors 2 , which in turn are easily accessible by bromination of $6 H-1,2$-oxazines $\mathbf{1}$. Lewis-acid promoted reaction of 1,2-oxazine $\mathbf{9 c}$ with 1-hexyne provided alkynyl-substituted pyridine derivative $\mathbf{1 2}$ thus demonstrating the potential of this approach for the synthesis of pyridines.
\end{abstract}

\section{Introduction}

A broad range of synthetic applications demonstrates that 1,2oxazine derivatives constitute a versatile class of N,O heterocycles [1-13]. Considerable attention has been paid to $6 H-1,2-$ oxazines 1 bearing a C-4,C-5-double bond [14-18], which are useful intermediates in the synthesis of $\gamma$-lactams [19], $\gamma$-amino acids [20], amino alcohols [20], aziridines [21], pyrrolizidines [22], and pyrrolidine derivatives $[15,23,24]$. In the context of our ongoing exploration of the synthetic potential of these heterocycles we were interested to modify the substitution pattern of the C-4,C-5 double bond of 6H-1,2-oxazines [25-27]. Herein, we describe our results dealing with the halogenation of
$6 H-1,2$-oxazines 1 and the use of the resulting products as precursors in palladium-catalyzed cross coupling reactions.

\section{Results and Discussion}

Not much is known about halogenated $6 H$-1,2-oxazines and only a few mostly inefficient procedures are described [28-32]. This prompted us to investigate a more practical access to halogenated $6 H$-1,2-oxazines. Gratifyingly, the desired 4-bromosubstituted $6 H$-1,2-oxazines $\mathbf{2 a - 2 c}$ could be prepared in a onepot procedure by bromine addition to precursors 1a-1c [14] and $\mathrm{HBr}$ elimination by treatment with triethylamine (Scheme 1). 
The 4-bromo-6H-1,2-oxazines were obtained in reasonable to good yields. The bromination of 3-phenyl-substituted $6 H-1,2-$ oxazine 1a often resulted in a mixture of several brominated products which are easily separable by chromatography. Depending on the reaction scale and the amount of bromine used ( 1.5 to 3 equiv) by-products such as $\mathbf{3 a}, \mathbf{4}$ and $\mathbf{5}$ could be isolated in varying yields. The unexpected formation of 4,5dibromo-6H-1,2-oxazine 3a can obviously be rationalized by addition of bromine to $\mathbf{2 a}$ and elimination of $\mathrm{HBr}$ during the bromination reaction of $\mathbf{1 a}$.

$$
\text { (us to }
$$

Scheme 1: Brominations of $6 \mathrm{H}-1,2$-oxazines. a) $\mathrm{Br}_{2}, \mathrm{Et}_{2} \mathrm{O},-30^{\circ} \mathrm{C}$, 2 h. b) $\mathrm{Et}_{3} \mathrm{~N},-30^{\circ} \mathrm{C}$ to r.t., overnight.

The literature describes just one related 4-chloro-substituted $6 H$-1,2-oxazine which was prepared by a hetero-Diels-Alder cycloaddition-elimination sequence of 2-chloro-1-nitroso-1phenyl-ethene and 1-bromo-2-ethoxyethene in low yield (22\%) [32]. As demonstrated in Scheme 2, a more efficient approach consists in chlorination of $6 H$-1,2-oxazines $\mathbf{1 a}, \mathbf{b}$ by addition of chlorine and subsequent base-induced dehydrochlorination. The expected 4-chloro-6H-1,2-oxazines $\mathbf{6 a}, \mathbf{b}$ were obtained in good yields. In analogy to the aforementioned bromination, the chlorination of 3-phenyl-6H-1,2-oxazine 1a also led to dihalogenation furnishing 4,5-dichloro-substituted compound $7 \mathbf{a}$ as a by-product in $13 \%$ yield.

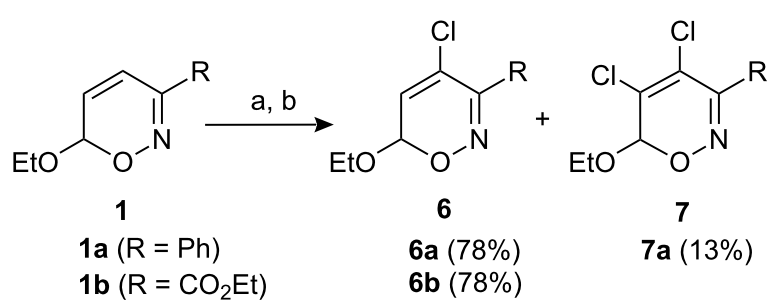

Scheme 2: Chlorinations of $6 \mathrm{H}-1,2$-oxazines. a) $\mathrm{Cl}_{2}, \mathrm{Et}_{2} \mathrm{O},-30^{\circ} \mathrm{C}$. b) $\mathrm{Et}_{3} \mathrm{~N},-30^{\circ} \mathrm{C}$ to r.t.
With the 4-halogenated $6 H$-1,2-oxazines 2 and $\mathbf{6}$ in hand, palladium-catalyzed cross couplings offer an efficient and useful approach for the synthesis of novel functionalized $6 H-1,2-$ oxazines. The Suzuki-coupling of the 4-bromo-substituted heterocycles $\mathbf{2 a}, \mathbf{b}$ with phenylboronic acid in the presence of $\mathrm{Pd}\left(\mathrm{PPh}_{3}\right)_{4}$ and sodium carbonate at $80{ }^{\circ} \mathrm{C}$ in toluene gave the expected 4-phenyl-substituted $6 H$-1,2-oxazines $8 \mathbf{a}$ or $\mathbf{8 b}$ in 82 and $77 \%$ yield (Scheme 3 ).

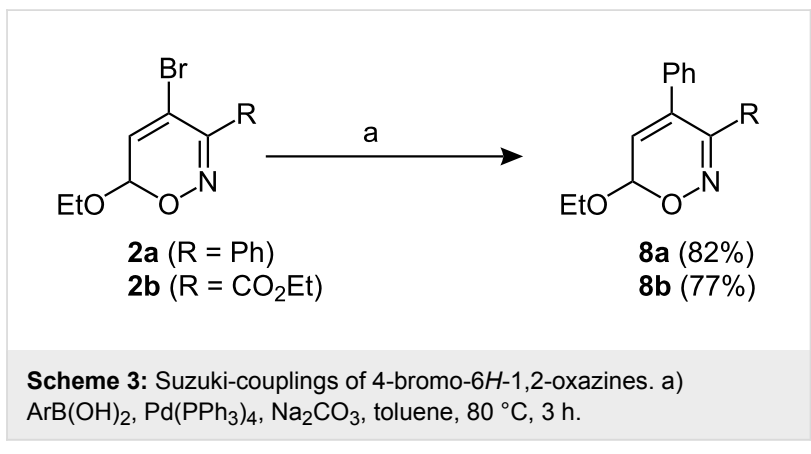

4-Bromo-6H-1,2-oxazine 2a also serves as suitable model substrate for Sonogashira-reactions (Scheme 4). When the coupling reaction of $\mathbf{2 a}$ with various terminal alkynes, such as phenylacetylene, trimethylsilylethyne and 1-hexyne, was performed under typical conditions $\left[\mathrm{PdCl}_{2}\left(\mathrm{PPh}_{3}\right)_{2}, \mathrm{CuI}, \mathrm{Et}_{3} \mathrm{~N}\right.$, toluene], the expected 4-alkynyl-substituted heterocycles 9a-9c were isolated in good yields. In contrast, when the same reaction conditions were applied to the coupling of $\mathbf{2 a}$ and methyl propargyl ether, product 9d was obtained only in very low yield. In addition, Sonogashira coupling of $\mathbf{2 a}$ and methyl propargyl ether performed by an alternative protocol $\left(\mathrm{Pd}(\mathrm{OAc})_{2}, \mathrm{CuI}, \mathrm{PPh}_{3}, \mathrm{NH} i \mathrm{Pr}_{2}\right.$ in DMF) afforded the expected product 9d and a byproduct bearing a 4-enyne moiety at 4-position. This indicates an addition of a second alkyne molecule to the primary product 9 . Similar results were observed for the Sonogashira reaction of 2a with propargylic alcohol [33].

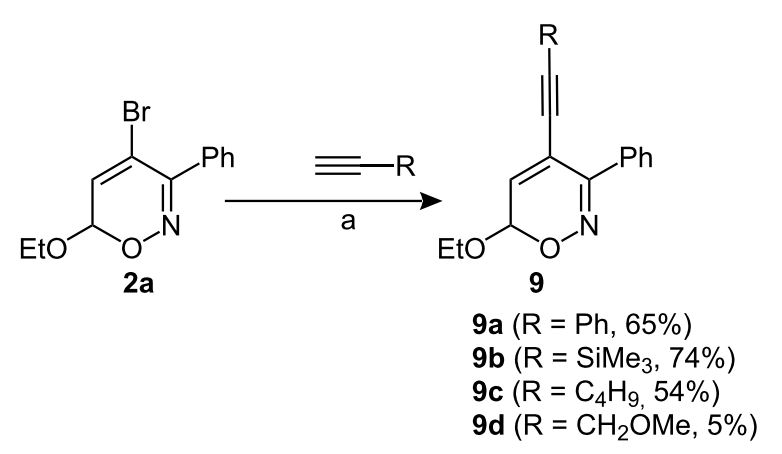

Scheme 4: Sonogashira-couplings of 4-bromo-6H-1,2-oxazines. a) $\mathrm{PdCl}_{2}\left(\mathrm{PPh}_{3}\right)_{2}$, Cul, $\mathrm{Et}_{3} \mathrm{~N}$, toluene, r.t., 6-20 h. 
After successful simple cross couplings of mono-halogenated 2 , the 4,5-dibromo-3-phenyl- $6 H$-1,2-oxazine 3a seemed to be an attractive candidate for a twofold Sonogashira reaction (Scheme 5). Treatment of $\mathbf{3 a}$ with an excess of phenylacetylene under conditions as described in Scheme 4 provided 5-bromo-4alkynyl-substituted $6 H$-1,2-oxazine 10a as single product in $65 \%$ yield. When the Sonogashira coupling was performed with trimethylsilylethyne under the same reaction conditions an inseparable 85:15-mixture of mono-alkynylated product 10b and bis-alkynylated compound $\mathbf{1 1 b}$ was obtained in reasonable yield. These reactions certainly deserve further optimization, however, they already show the potential of compounds such as 3a to serve as precursors for two subsequent coupling reactions.

$$
\text { (65\% 10a), } \operatorname{SiMe}_{3}(66 \% ; \mathbf{1 0 b}: 11 \mathbf{b}=85: 15)
$$

Scheme 5: Sonogashira-couplings of 4,5-dibromo-6H-1,2-oxazines. a) $\mathrm{PdCl}_{2}\left(\mathrm{PPh}_{3}\right)_{2}, \mathrm{Cul}_{3} \mathrm{Et}_{3} \mathrm{~N}$, toluene, r.t., $4 \mathrm{~h}$ to overnight.

\section{Conclusion and Perspective}

In conclusion, we have successfully demonstrated that a series of 4-aryl- and 4-alkynyl-substituted $6 H$-1,2-oxazines $\mathbf{8}, \mathbf{9}$, and $\mathbf{1 0}$ are easily accessible in short reaction sequences starting from precursors 1 . These $6 H-1,2$-oxazines should allow access to many interesting five- and six-membered heterocycles. As illustrated in Scheme 6, the 4-hex-1-ynyl-3-phenyl-6H-1,2oxazine 9c can be converted into the trisubstituted pyridine derivative $\mathbf{1 2}$ by treatment of $9 \mathbf{c}$ with boron trifluoride etherate in the presence of an excess of 1-hexyne via an azapyrylium intermediate $[34,35]$. Additional investigations are required to optimize the preparation diynes of type 11. Conversion of the new functionalized $6 H$-1,2-oxazines to highly substituted pyridine derivatives will also be reported in due course.

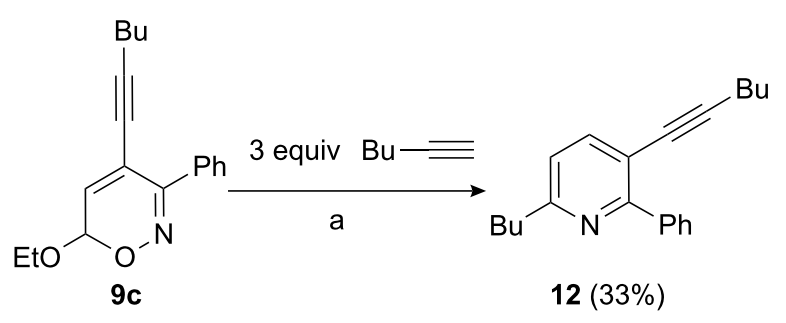

Scheme 6: Preparation of trisubstituted pyridine derivatives: a) $\mathrm{BF}_{3} \cdot \mathrm{OEt}_{2}, \mathrm{CH}_{2} \mathrm{Cl}_{2},-78{ }^{\circ} \mathrm{C}$ to r.t., overnight.

\section{Experimental \\ Bromination of $6 \mathrm{H}-1,2-$ oxazine 1a, typical procedure}

$6 H$-1,2-Oxazine 1a $(5.35 \mathrm{~g}, 26.3 \mathrm{mmol})$ was dissolved in diethyl ether $(200 \mathrm{~mL})$ and treated with bromine $(2.75 \mathrm{~mL}, 53.7$ $\mathrm{mmol})$ at $-30{ }^{\circ} \mathrm{C}$ under argon atmosphere. After $2 \mathrm{~h} \mathrm{Et}_{3} \mathrm{~N}(54.0$ $\mathrm{mL}, 390 \mathrm{mmol}$ ) was added. The reaction mixture was warmed to r.t. overnight and quenched with water $(100 \mathrm{~mL})$. The aqueous phase was extracted with $\mathrm{CH}_{2} \mathrm{Cl}_{2}(2 \times 50 \mathrm{~mL})$ and the combined organic phases were dried with $\mathrm{Na}_{2} \mathrm{SO}_{4}$. Purification of the crude product by column chromatography $\left(\mathrm{SiO}_{2}\right.$, hexane:EtOAc 8:1, then 4:1) gave the 4-bromo-substituted $6 H$-1,2-oxazine $2 \mathrm{a}(5.11 \mathrm{~g}, 69 \%)$, the 4,5-dibromo-substituted by-product 3a $(0.821 \mathrm{~g}, 9 \%)$, and starting material 1a $(0.335 \mathrm{~g}$, $6 \%$ ).

4-Bromo-6-ethoxy-3-phenyl-6H-1,2-oxazine (2a): yellow-brown oil. ${ }^{1} \mathrm{H} \mathrm{NMR}\left(\mathrm{CDCl}_{3}, 300 \mathrm{MHz}\right): \delta=1.23(\mathrm{t}, J=$ $\left.7.1 \mathrm{~Hz}, 3 \mathrm{H}, \mathrm{CH}_{3}\right), \mathrm{AB}$ part of $\mathrm{ABX}_{3}$ system $\left(\delta_{\mathrm{A}}=3.68, \delta_{\mathrm{B}}=\right.$ $\left.3.95, J_{\mathrm{AX}}=J_{\mathrm{BX}}=7.1 \mathrm{~Hz}, J_{\mathrm{AB}}=9.8 \mathrm{~Hz}, 2 \mathrm{H}, \mathrm{OCH}_{2}\right), 5.58(\mathrm{~d}, J$ $=5.2 \mathrm{~Hz}, 1 \mathrm{H}, 6-\mathrm{H}), 6.70(\mathrm{~d}, J=5.2 \mathrm{~Hz}, 1 \mathrm{H}, 5-\mathrm{H}), 7.35-7.50$, 7.50-7.60 (2 m, $3 \mathrm{H}, 2 \mathrm{H}, \mathrm{Ph}) \mathrm{ppm} .{ }^{13} \mathrm{C} \mathrm{NMR}\left(\mathrm{CDCl}_{3}, 75.5\right.$ $\mathrm{MHz}): \delta=14.8\left(\mathrm{q}, \mathrm{CH}_{3}\right), 64.3\left(\mathrm{t}, \mathrm{OCH}_{2}\right), 94.7$ (d, C-6), 112.9 (s, C-4), 127.8, 128.0, 128.8, 129.7, 133.1 (4 d, s, Ph, C-5), 156.2 (s, C-3) ppm. For the complete characterization, see ref. [31].

4,5-Dibromo-6-ethoxy-3-phenyl-6H-1,2-oxazine (3a): brown oil. ${ }^{1} \mathrm{H} \mathrm{NMR}\left(\mathrm{CDCl}_{3}, 300 \mathrm{MHz}\right): \delta=1.25(\mathrm{t}, J=7.1 \mathrm{~Hz}, 3 \mathrm{H}$, $\left.\mathrm{CH}_{3}\right), \mathrm{AB}$ part of $\mathrm{ABX}_{3}$ system $\left(\delta_{\mathrm{A}}=3.76, \delta_{\mathrm{B}}=3.97, J_{\mathrm{AX}}=\right.$ $\left.J_{\mathrm{BX}}=7.1 \mathrm{~Hz}, J_{\mathrm{AB}}=9.7 \mathrm{~Hz}, 2 \mathrm{H}, \mathrm{OCH}_{2}\right), 5.71(\mathrm{~s}, 1 \mathrm{H}, 6-\mathrm{H})$, 7.38-7.48, 7.49-7.56 (2 m, 3 H, $2 \mathrm{H}, \mathrm{Ph}) \mathrm{ppm} .{ }^{13} \mathrm{C} \mathrm{NMR}$ $\left(\mathrm{CDCl}_{3}, 75.5 \mathrm{MHz}\right): \delta=14.7\left(\mathrm{q}, \mathrm{CH}_{3}\right), 65.0\left(\mathrm{t}, \mathrm{OCH}_{2}\right), 99.8(\mathrm{~d}$, C-6), 114.4, 124.8 (2 s, C-4, C-5), 128.1, 128.9, 129.9, 133.3 (3 d, s, Ph), 155.9 (s, C-3) ppm. IR (neat): 3065-2900 (=C-H, $\mathrm{C}-\mathrm{H}), 1630(\mathrm{C}=\mathrm{N}), 1600(\mathrm{C}=\mathrm{C}) \mathrm{cm}^{-1}$. HRMS $\left(80 \mathrm{eV}, 40{ }^{\circ} \mathrm{C}\right)$ $m / z$ calcd for $\mathrm{C}_{12} \mathrm{H}_{11}{ }^{79} \mathrm{Br}_{2} \mathrm{NO}_{2}$ : 358.9157; found: 358.9160 .

\section{Chlorination of $6 H-1,2-o x a z i n e ~ 1 b$, typical procedure}

Chlorine gas was passed into diethyl ether $(28 \mathrm{~mL})$ at $-30{ }^{\circ} \mathrm{C}$ until the solution became dark yellow. Then, $6 H$-1,2-oxazine $\mathbf{1 b}$ $(0.200 \mathrm{~g}, 1.00 \mathrm{mmol})$ was added and the reaction mixture was monitored by TLC; upon complete consumption, triethylamine (2.00 $\mathrm{mL}, 27.8 \mathrm{mmol}$ ) was added at $-30^{\circ} \mathrm{C}$ and the mixture was slowly warmed to r.t. After addition of brine, the phases were separated, the aqueous phase was extracted with $\mathrm{CH}_{2} \mathrm{Cl}_{2}(2 \times$ $20 \mathrm{~mL}$ ) and the combined organic phases were dried with $\mathrm{Na}_{2} \mathrm{SO}_{4}$. Column chromatography $\left(\mathrm{SiO}_{2}\right.$, hexane, hexane:EtOAc 9:1, then 4:1) afforded the 4-chloro-substituted product $6 \mathbf{b}(0.182 \mathrm{~g}, 78 \%)$ as pale-yellow oil. 
Ethyl 4-chloro-6-ethoxy-6H-1,2-oxazine-3-carboxylate $(\mathbf{6 b}):{ }^{1} \mathrm{H}$ NMR $\left(\mathrm{CDCl}_{3}, 300 \mathrm{MHz}\right): \delta=1.22\left(\mathrm{t}, J=7.1 \mathrm{~Hz}, 3 \mathrm{H}, \mathrm{CH}_{3}\right)$, $1.40\left(\mathrm{t}, J=7.2 \mathrm{~Hz}, 3 \mathrm{H}, \mathrm{CH}_{3}\right), \mathrm{AB}$ part of $\mathrm{ABX}_{3}$ system $\left(\delta_{\mathrm{A}}=\right.$ $3.68, \delta_{\mathrm{B}}=3.95, J_{\mathrm{AX}}=J_{\mathrm{BX}}=7.1 \mathrm{~Hz}, J_{\mathrm{AB}}=9.6 \mathrm{~Hz}, 2 \mathrm{H}$, $\left.\mathrm{OCH}_{2}\right), 4.40$ (q, $\left.J=7.2 \mathrm{~Hz}, 2 \mathrm{H}, \mathrm{OCH}_{2}\right), 5.72(\mathrm{~d}, J=5.0 \mathrm{~Hz}, 1$ $\mathrm{H}, 6-\mathrm{H}), 6.34(\mathrm{~d}, J=5.0 \mathrm{~Hz}, 1 \mathrm{H}, 5-\mathrm{H}) \mathrm{ppm} .{ }^{13} \mathrm{C} \mathrm{NMR}\left(\mathrm{CDCl}_{3}\right.$, $75.5 \mathrm{MHz}$ ): $\delta=13.9,14.7\left(2 \mathrm{q}, \mathrm{CH}_{3}\right), 62.5,64.6\left(2 \mathrm{t}, \mathrm{OCH}_{2}\right)$, 95.3 (d, C-6), 121.1 (s, C-4), 122.7 (d, C-5), 148.5 (s, C-3), 160.3 (s, C=O) ppm. IR (neat): 3105-2975 (=C-H, C-H), 1745 $(\mathrm{C}=\mathrm{O}), 1615(\mathrm{C}=\mathrm{N}) \mathrm{cm}^{-1} \cdot \mathrm{C}_{9} \mathrm{H}_{12} \mathrm{ClNO}_{4}$ (233.7): calcd. $\mathrm{C}$, 46.27; H, 5.18; N, 5.99; found: C, 46.35; H, 5.16; N, 6.08 .

\section{Suzuki-coupling of 4-bromo-substituted $6 H-1,2-o x a z i n e ~ 2 a$, typical procedure}

$6 H$-1,2-Oxazine 2a (0.0935 g, $0.33 \mathrm{mmol})$, phenylboronic acid $(0.122 \mathrm{~g}, 1.00 \mathrm{mmol})$ and $\mathrm{Pd}\left(\mathrm{PPh}_{3}\right)_{4}(0.016 \mathrm{~g}, 0.0138 \mathrm{mmol})$ were dissolved in a mixture of toluene/ $\mathrm{MeOH}(3 \mathrm{~mL} / 0.75 \mathrm{~mL})$ in a heat-gun-dried and argon-flushed flask. A $2 \mathrm{M} \mathrm{Na}_{2} \mathrm{CO}_{3}$ solution $(1.5 \mathrm{~mL})$ was finally added and the reaction mixture was heated for $15 \mathrm{~h}$ at $80{ }^{\circ} \mathrm{C}$. Then, the reaction mixture was cooled to r.t. and washed with $2 \mathrm{M} \mathrm{Na}_{2} \mathrm{CO}_{3}$ (with $1 \% \mathrm{NH}_{3}$ ) solution. After separation of the phases, the aqueous phase was extracted with $\mathrm{CH}_{2} \mathrm{Cl}_{2}(3 \times 5 \mathrm{~mL})$ and the combined organic phases were dried with $\mathrm{Na}_{2} \mathrm{SO}_{4}$. The crude product was purified by column chromatography $\left(\mathrm{SiO}_{2}\right.$, hexane:EtOAc 9:1, then $4: 1)$ to afford the Suzuki product $8 \mathbf{a}(0.076 \mathrm{~g}, 82 \%)$ as a pale-yellow solid, mp $68-70{ }^{\circ} \mathrm{C}$.

6-Ethoxy-3,4-diphenyl-6H-1,2-oxazine (8a): ${ }^{1} \mathrm{H} \mathrm{NMR}\left(\mathrm{CDCl}_{3}\right.$, $300 \mathrm{MHz}): \delta=1.25\left(\mathrm{t}, J=7.1 \mathrm{~Hz}, 3 \mathrm{H}, \mathrm{CH}_{3}\right), \mathrm{AB}$ part of $\mathrm{ABX}_{3}$ system $\left(\delta_{\mathrm{A}}=3.75, \delta_{\mathrm{B}}=4.01, J_{\mathrm{AX}}=J_{\mathrm{BX}}=7.1 \mathrm{~Hz}, J_{\mathrm{AB}}=9.8\right.$ $\left.\mathrm{Hz}, 2 \mathrm{H}, \mathrm{OCH}_{2}\right), 5.73$ (d, $\left.J=4.9 \mathrm{~Hz}, 1 \mathrm{H}, 6-\mathrm{H}\right), 6.37$ (d, $J=4.9$ $\mathrm{Hz}, 1 \mathrm{H}, 5-\mathrm{H}), 7.05-7.10,7.15-7.27,7.30-7.35$ (3 m, 4 H, 4 H, $2 \mathrm{H}, \mathrm{Ph}) \mathrm{ppm} .{ }^{13} \mathrm{C} \mathrm{NMR}\left(\mathrm{CDCl}_{3}, 75.5 \mathrm{MHz}\right): \delta=15.0$ (q, $\left.\mathrm{CH}_{3}\right), 64.2\left(\mathrm{t}, \mathrm{OCH}_{2}\right), 93.0$ (d, C-6), 124.0 (d, C-5), 128.0, $128.2,128.4,128.7,129.0,130.2,133.9,136.5(5 \mathrm{~d}, 3 \mathrm{~s}, \mathrm{Ph}$, C-4), 157.7 (s, C-3) ppm. IR (KBr): 3040-2930 (=C-H, C-H), $1620(\mathrm{C}=\mathrm{N}), 1600(\mathrm{C}=\mathrm{C}) \mathrm{cm}^{-1} \cdot \mathrm{C}_{18} \mathrm{H}_{17} \mathrm{NO}_{2}$ (279.3): calcd. $\mathrm{C}$, 77.39; H, 6.13; N, 5.01; found: C, 77.82; H, 6.37; N, 5.08.

\section{Sonogashira-coupling of 4-bromo-substi- tuted $6 \mathrm{H}-1,2-$ oxazine $\mathbf{2 a}$, typical procedure}

6H-1,2-Oxazine 2a (0.850 g, $3.19 \mathrm{mmol})$, trimethylsilylethyne (0.87 mL, $6.17 \mathrm{mmol}), \mathrm{PdCl}_{2}\left(\mathrm{PPh}_{3}\right)_{2}(0.114 \mathrm{~g}, 0.16 \mathrm{mmol}), \mathrm{CuI}$ $(0.019 \mathrm{~g}, 0.10 \mathrm{mmol})$ and $\mathrm{Et}_{3} \mathrm{~N}(1.3 \mathrm{~mL})$ were dissolved in toluene $(15 \mathrm{~mL})$ in a heat-gun-dried and argon-flushed flask and the reaction mixture was stirred at r.t. for $20 \mathrm{~h}$. The reaction mixture was quenched with water $(5 \mathrm{~mL})$. The aqueous phase was extracted with $\mathrm{CH}_{2} \mathrm{Cl}_{2}(3 \times 10 \mathrm{~mL})$ and the combined organic phases were dried with $\mathrm{Na}_{2} \mathrm{SO}_{4}$. Purification of the crude product by column chromatography $\left(\mathrm{SiO}_{2}\right.$, hexane:
EtOAc 20:1, then 4:1) afforded the 4-alkynyl-substituted $6 H$-1,2-oxazine $9 \mathbf{b}(0.711 \mathrm{~g}, 74 \%)$ as a colorless oil.

6-Ethoxy-3-phenyl-4-(trimethylsilylethynyl)-6H-1,2-oxazine (9b): ${ }^{1} \mathrm{H} \mathrm{NMR}\left(\mathrm{CDCl}_{3}, 250 \mathrm{MHz}\right): \delta=0.71\left(\mathrm{~s}, 9 \mathrm{H}, \mathrm{SiMe}_{3}\right)$, $1.21\left(\mathrm{t}, J=7.1 \mathrm{~Hz}, 3 \mathrm{H}, \mathrm{CH}_{3}\right), \mathrm{AB}$ part of $\mathrm{ABX}_{3}$ system $\left(\delta_{\mathrm{A}}=\right.$ $3.68, \delta_{\mathrm{B}}=3.96, J_{\mathrm{AX}}=J_{\mathrm{BX}}=7.1 \mathrm{~Hz}, J_{\mathrm{AB}}=9.7 \mathrm{~Hz}, 2 \mathrm{H}$, $\left.\mathrm{OCH}_{2}\right), 5.62(\mathrm{~d}, J=5.1 \mathrm{~Hz}, 1 \mathrm{H}, 6-\mathrm{H}), 5.69(\mathrm{~d}, J=5.1 \mathrm{~Hz}, 1 \mathrm{H}$, 5-H), 7.34-7.44, 7.67-7.73 (2 m, $3 \mathrm{H}, 2 \mathrm{H}, \mathrm{Ph}) \mathrm{ppm} .{ }^{13} \mathrm{C}$ NMR $\left(\mathrm{CDCl}_{3}, 125.8 \mathrm{MHz}\right): \delta=-0.7\left(\mathrm{q}, \mathrm{SiMe}_{3}\right), 14.9\left(\mathrm{q}, \mathrm{CH}_{3}\right), 64.2$ $\left(\mathrm{t}, \mathrm{OCH}_{2}\right), 92.0$ (d, C-6), 99.5, 101.9 (2 s, C $\left.\equiv \mathrm{C}\right), 114.0$ (s, C-4), 127.7, 128.7, 129.5, 130.1, 132.9 (4 d, s, Ph, C-5), 155.5 (s, C-3) ppm. IR (neat): 3085-2900 (=C-H, C-H), $2160(\mathrm{C} \equiv \mathrm{C})$, $1620(\mathrm{C}=\mathrm{C}), 1580(\mathrm{C}=\mathrm{N}) \mathrm{cm}^{-1} \cdot \mathrm{C}_{17} \mathrm{H}_{21} \mathrm{NO}_{2} \mathrm{Si}$ (299.5): calcd. C, 68.19; H, 7.07; N, 4.68; found: C, 68.17; H, 7.08; N, 4.74.

\section{Acknowledgments}

Generous support by the Deutsche Forschungsgemeinschaft (Graduiertenkolleg at the Technische Universität Dresden "Struktur-Eigenschafts-Beziehungen bei Heterocyclen"), the Fonds der Chemischen Industrie and the Bayer-Schering Pharma AG is most gratefully acknowledged. We also thank Luise Schefzig and Ute Hain for their experimental assistance.

\section{References}

1. Gilchrist, T. L. Chem. Soc. Rev. 1983, 12, 53-73. doi:10.1039/cs9831200053

2. Tsoungas, P. G. Heterocycles 2002, 57, 915-953. doi:10.3987/REV-02-548

3. Reissig, H.-U.; Zimmer, R. In Science of Synthesis Houben-Weyl Methods of Molecular Transformations; Trost, B. M.; Molander, G. A., Eds.; Thieme: Stuttgart, Germany, 2006; Vol. 33, pp 371-389.

4. Lyapkalo, I. M.; loffe, S. L. Russ. Chem. Rev. (engl. Transl.) 1998, 67, 467-485.

5. Tishkov, A. A.; Reissig, H.-U.; loffe, S. L. Synlett 2002, 863-866. doi:10.1055/s-2002-31908

6. Young, I. S.; Kerr, M. A. Angew. Chem. 2003, 115, 3131-3134. Angew. Chem. Int. Ed. 2003, 42, 3023-3026. doi:10.1002/anie.200351573

7. Helms, M.; Schade, W.; Pulz, R.; Watanabe, T.; Al-Harrasi, A.; Fisera, L.; Hlobilova, I.; Zahn, G.; Reissig, H.-U. Eur. J. Org. Chem. 2005, 1003-1019. doi:10.1002/ejoc.200400627

8. Cardona, F.; Goti, A. Angew. Chem. 2005, 117, 8042-8045. Angew. Chem. Int. Ed. 2005, 44, 7832-7835. doi:10.1002/anie.200502640

9. Sibi, M. P.; Ma, Z.; Jasperse, C. P. J. Am. Chem. Soc. 2005, 127, 5764-5765. doi:10.1021/ja0421497

10. Kumarn, S.; Shaw, D. M.; Ley, S. V. Chem. Commun. 2006, 3211-3213. doi:10.1039/b606338a

11. Lu, C.-D.; Zakarian, A. Angew. Chem. 2008, 120, 6935-6937. Angew. Chem. Int. Ed. 2008, 47, 6829-6831. doi:10.1002/anie.200801652

12. Brasholz, M.; Reissig, H.-U.; Zimmer, R. Acc. Chem. Res. 2009, 42 , 45-56. doi:10.1021/ar800011h 
13. Sukhorukov, A. Yu.; Lesiv, A. V.; Khomutova, Y. A.; loffe, S. L. Synthesis 2009, 741-754. doi:10.1055/s-0028-1083360

14. Homann, K.; Angermann, J.; Collas, M.; Zimmer, R.; Reissig, H.-U. J. Prakt. Chem. 1998, 340, 649-655. doi:10.1002/prac.19983400709

15. Zimmer, R.; Orschel, B.; Scherer, S.; Reissig, H.-U. Synthesis 2002, 1553-1563. doi:10.1055/s-2002-33328

16. Zimmer, R.; Reissig, H.-U. Angew. Chem. 1988, 100, 1576-1577. Angew. Chem., Int. Ed. Engl. 1988, 27, 1518-1519. doi:10.1002/anie.198815181

17. Zimmer, R.; Reissig, H.-U. Liebigs Ann. Chem. 1991, 553-562. doi:10.1002/jlac.1991199101101

18. Zimmer, R.; Reissig, H.-U. J. Org. Chem. 1992, 57, 339-347. doi:10.1021/jo00027a058

19. Zimmer, R.; Reissig, H.-U.; Lindner, H. J. Liebigs Ann. Chem. 1992, 621-624. doi:10.1002/jlac.1992199201106

20. Zimmer, R.; Hoffmann, M.; Reissig, H.-U. Chem. Ber. 1992, 125, 2243-2248. doi:10.1002/cber.19921251012

21. Zimmer, R.; Homann, K.; Reissig, H.-U. Liebigs Ann. Chem. 1993, 1155-1157. doi:10.1002/jlac.1993199301186

22. Zimmer, R.; Collas, M.; Czerwonka, R.; Hain, U.; Reissig, H.-U. Synthesis 2008, 237-244. doi:10.1055/s-2007-990946

23. Buchholz, M.; Reissig, H.-U. Eur. J. Org. Chem. 2003, 3524-3533. doi:10.1002/ejoc.200300234

24. Reissig, H.-U.; Homann, K.; Hiller, F.; Zimmer, R. Synthesis 2007, 2681-2689. doi:10.1055/s-2007-983802

25. Zimmer, R.; Homann, K.; Angermann, J.; Reissig, H.-U. Synthesis 1999, 1223-1235. doi:10.1055/s-1999-3524

26. Buchholz, M.; Reissig, H.-U. Synthesis 2002, 1412-1422. doi:10.1055/s-2002-33107

27. Schmidt, E.; Reissig, H.-U.; Zimmer, R. Synthesis 2006, 2074-2084. doi:10.1055/s-2006-942403

28. Birkofer, L.; Feldmann, H. Liebigs Ann. Chem. 1964, 677, 150-153. doi:10.1002/jlac.19646770121

29. Abramovitch, R. A.; Shinkai, I.; Cue, B. W.; Ragan, F. A.; Atwood, J. C. J. Heterocycl. Chem. 1976, 13, 415-417. doi:10.1002/jhet.5570130247

30. Baird, M. S.; Li, X.; Al-Dulayymi, J. R.; Kurdjukov, A. I.; Pavlov, V. A. J. Chem. Soc., Perkin Trans. 1 1993, 2507-2508. doi:10.1039/P19930002507

31. Paulini, K.; Reissig, H.-U. Chem. Ber. 1994, 127, 685-689. doi:10.1002/cber.19941270418

32. Zimmer, R.; Angermann, J.; Hain, U.; Hiller, F.; Reissig, H.-U. Synthesis 1997, 1467-1474. doi:10.1055/s-1997-1373

33. Duhs, M.-A. Bachelor Thesis, Freie Universität Berlin, 2006.

34. Zimmer, R.; Reissig, H.-U.; Homann, K. J. Prakt. Chem. 1995, 337, 521-528. doi:10.1002/prac. 199533701112

35. Homann, K.; Zimmer, R.; Reissig, H.-U. Heterocycles 1995, 40, 531-537. doi:10.3987/COM-94-S77

\section{License and Terms}

This is an Open Access article under the terms of the Creative Commons Attribution License

(http://creativecommons.org/licenses/by/2.0), which permits unrestricted use, distribution, and reproduction in any medium, provided the original work is properly cited.

The license is subject to the Beilstein Journal of Organic Chemistry terms and conditions:

(http://www.beilstein-journals.org/bjoc)

The definitive version of this article is the electronic one which can be found at:

doi:10.3762/bjoc.5.44 